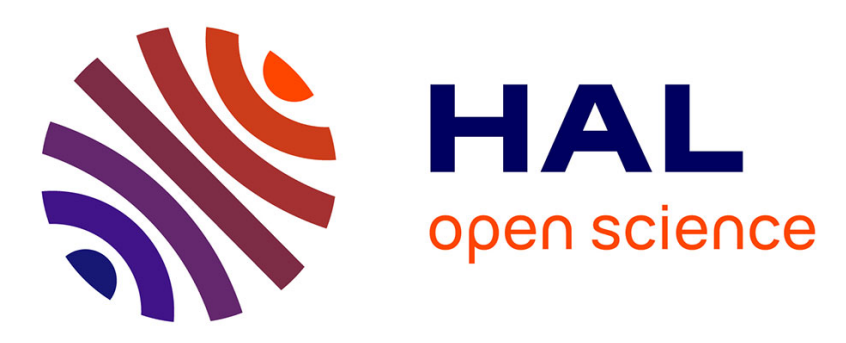

\title{
Eye Gaze Sequence Analysis to Model Memory in E-education
}

Maël Beuget, Sylvain Castagnos, Christophe Luxembourger, Anne Boyer

\section{To cite this version:}

Maël Beuget, Sylvain Castagnos, Christophe Luxembourger, Anne Boyer. Eye Gaze Sequence Analysis to Model Memory in E-education. 20th international conference on Artificial Intelligence in Education (AIED 2019), Jun 2019, Chicago, United States. hal-02471999

\section{HAL Id: hal-02471999 https://hal.science/hal-02471999}

Submitted on 9 Feb 2020

HAL is a multi-disciplinary open access archive for the deposit and dissemination of scientific research documents, whether they are published or not. The documents may come from teaching and research institutions in France or abroad, or from public or private research centers.
L'archive ouverte pluridisciplinaire HAL, est destinée au dépôt et à la diffusion de documents scientifiques de niveau recherche, publiés ou non, émanant des établissements d'enseignement et de recherche français ou étrangers, des laboratoires publics ou privés. 


\title{
Eye Gaze Sequence Analysis to Model Memory in E-education
}

\author{
M. Beuget ${ }^{1}$, S. Castagnos ${ }^{1}$, C. Luxembourger ${ }^{2}$, and A. Boyer ${ }^{1}$ \\ 1 CNRS-LORIA-University of Lorraine \\ 2 2LPN-University of Lorraine
}

\begin{abstract}
Intelligent Tutoring Systems are now mature technologies that successfully help students to acquire new knowledge and competencies through various educational methods and in a personalized way. However, evaluating precisely what they recall at the end of the learning process remains a complex task. In this paper, we study if there are correlations between memory and gaze data in the context of e-education. Our long-term goal is to model the memory of students thank to an eyetracker in a continuous and transparent way. These models could then be used to adapt recommendations of pedagogical resources to the students learning rate. So as to address this research question, we designed an experiment where students were asked to learn a short lesson about Esperanto. Our results show that some gaze characteristics are correlated with recall in memory.
\end{abstract}

Keywords: Eye-tracking study $\cdot$ memory $\cdot$ gaze behavior $\cdot$ e-education

\section{Introduction}

The introduction of digital technologies in the society during the past decades impacts inevitably many aspects of our lives. Education is one of them, and the way numeric tools can improve the quality of learning is nowadays a fullfledged research field. Intelligent Tutoring Systems (ITS) are pieces of software that help students at mastering courses. They provide them with some lessons, incorporate knowledge assessment tools, allow instant and personalized feedback, and eventually propose students to train on their specific academic deficiencies. However, estimating accurately a user state of knowledge remains a difficult task. It is quite impossible to cover every aspect of the lesson through an exam, as the evaluation process should not be too much time consuming. In some cases, students may also provide randomly correct answers. An imprecise or incomplete user model may impact the feedback quality and the relevance of the recommendations provided by ITS. To overcome this problem, we are wondering if it is possible to infer more precisely what the user remembers by collecting implicit traces of interactions. The latter are not conscientiously indicated by the user, and could be for example action logs, facial expressions or eye movements. Gaze data interests us more specifically as it has been reported that the gaze behavior could reflect some cognitive processes $[3,13]$. Our goal is to analyze if 
it could exist correlations between gaze characteristics and the fact to remember some courses items. To do so, we designed a pilot study where 22 students had to learn a short lesson about Esperanto while wearing eye-tracking glasses. After the learning phase, they were asked to pass an exam. We then compared the gaze data with their grade on the exam. Results show that fixation durations, scanpath length and scanpath angles are good predictors of what have been recalled. As a perspective, eye-trackers are thus promising tools to model users' memory in real time when they are reading their lessons.

\section{Related work}

\subsection{A brief history of the memory models}

It exists not only one but several forms of memories, and the way we can model these forms of memories is not unanimous among psychologists. The most widely accepted models are those proposed by Atkinson-Shiffrin [12], Baddeley [2] and Miyake et al. [14]. In each of them, memory refers to the ability to encode, store and retrieve past experiences. It is composed of several memory modules. The sensory register allows the incoming information to be encoded for treatment. The short-term memory holds a small amount of information in mind in an active readily available state for a few seconds. The working memory manages and handles the information required to carry out complex tasks such as comprehension, reasoning, and learning. The differences between the models of Atkinson-Shiffrin, Baddeley and Miyake mainly focus on the distinction and overlap between short-term memory and working memory [1]. Finally, the longterm memory stores information for an extended period of time, consciously or not [17]. In our case, we take an interest in the working memory since it is the one involved in the learning process.

In parallel with this segmentation of the different forms of memory, researchers distinguish recognition and recall. The recognition process is the fact to remember something when the stimuli is present, whereas the recall involves to remember a stimulus which is not physically present [6]. In the context of e-education, we assume that learning a lesson correctly consists in storing the information in the recall memory.

\subsection{Linking cognitive processes and eye movements}

According to Just and Carpenter, what a person is looking at is assumed to indicate the on top of the stack thought of cognitive processes [9]. More recently, Steichen et al. aimed at identifying gaze patterns [18], while Bondareva et al. use the gaze data of users while they interact with an intelligent tutoring system to predict in real time the efficiency of the users learning process [3]. However, they did not investigate the possibility to infer memory. Let us note that memorized items are the results of the learning process. Predicting the quality of learning therefore amounts to predicting the amount of information stored, without however knowing how to distinguish the forgotten pieces of information from the 
remembered ones. Regarding the link between memory and gaze data, Hanula et al. have established the ability to predict recognition through a user study where the stimuli proposed to users were faces of people [7]. Several studies also tried to exploit gaze characteristics to predict memorability [4] and recall [13] of images. To our knowledge, no study has sought to establish this link between gaze and recall in a context closer to e-education, i.e. with multimedia content (text and images). This is what drives our research project. Our purpose is to verify if a link could exists between the recall process and some gaze metrics, to later potentially infer a user state of memories.

To analyze gaze data the first step is to transform the eye-tracker's sampled signal into a scanpath composed by fixations and saccades. Fixations are points of gaze where the fovea is concentrated for a short period of about $200 \mathrm{~ms}$, whereas saccades are the eye movement that link two consecutive fixations. Many algorithms are described in the literature to transform the sampled signal into a scanpath $[15,10,11]$. In our study we used the IV-T algorithm provided by the Tobii Pro lab software. The way we can extract metrics and information from scanpath has also been widely documented $[8,5,16,13]$.

\section{User study}

We designed a pilot study where subjects were asked to learn a short lesson ( 5 printed static pages from an online course) and had to report their knowledge during an exam. Documents were not allowed during this exam. We have chosen to work in the context of language learning. So as to avoid the bias of languages already learned, we based our lesson on the Esperanto language. The latter has been created in 1887 with the ambition to become a vehicular language, and has been built by combining several existing languages. It is one of the less taught language, so few people studied Esperanto. We had 22 participants (10 females / 12 males) aged from 11 to 16 year old. Our eye-tracker was a Tobii Pro Glasses 2 (100Hz frequency rate). The experiment is divided in 5 parts and lasts about 1 hour (see Figure 1): the first part is the vocabularys WISC subtest, the second part is about to learn the Esperanto lesson, the third part is the codes WISC subtest, the forth part is a quiz about the lesson, and the last part is the letter-number sequencings WISC subtest. WISC stands for Wechsler Intelligence Scale for Children and is the most widely used IQ test for children (especially in the US, Canada, and Europe). It is composed by several subtests, each of them estimating precise cognitive abilities. The vocabulary subtest estimates the verbal comprehension, fluency and word-knowing, the code part estimates a learning factor and the non-verbal working memory speed, and the letter-number sequencing subtest estimates the verbal auditory recall (working memory). The second and forth part of the experiment (lesson + exam) are about to estimate a learning factor and the recall memory. The participants are evaluated on the translation of words and sentences, on the differences between the two alphabets, and on some elements of the grammar. We chose to use the WISC subtests at distracting tasks to limit the primacy-recency bias. 


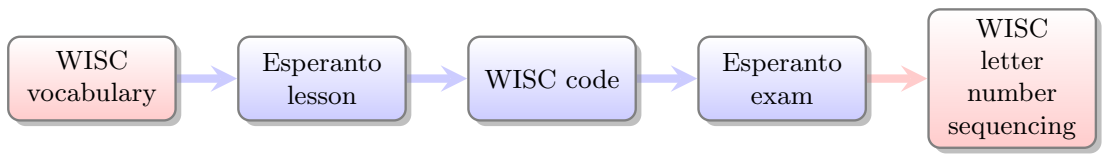

Fig. 1. The 5 parts of our experiment: blue ones are recorded with the eye-tracker.

\section{Analysis}

Many variables have been collected through our experiment: the WISC subtests score, the gaze data, the results of the exam. We studied the distribution of the gaze data with the Shapiro-Wilk test, and found that some gaze characteristics do not follow the theoretical normal distribution. We thus chose to use an ANOVA permutation test as it is a non-parametric test. We considered 21 global gaze metrics including the normalized sum, mean and standard deviation of fixation duration, saccade horizontal amplitude, vertical amplitude, vectorial amplitude, absolute and relative angles, and pupil dilatation as defined in [8, 13]. We used the aovp() $\mathrm{R}$ function from the lmperm package to perform our tests. We tried to explain the Esperanto test score or the WISC subtest scores by finding an interesting combination of factors (gaze metrics). Due to the combinatorial complexity we tested up to 6 factors at the same time and saved the models when all the factors were significant ( $\mathrm{p}$-value $<0.05$ ). Figure 2 shows an example of gaze features that are highly correlated to the global exam score.

\begin{tabular}{|l|l|l|l|l|l|}
\hline & Df & R Sum Sq & R Mean Sq & Iter & Pr(Prob) \\
\hline MedianFixationDuration & 1 & 351.17 & 351.17 & 5000 & $<2 \mathrm{e}-16^{* * *}$ \\
\hline ScanpathLengthNorm & 1 & 204.33 & 204.33 & 5000 & $0.0026^{* *}$ \\
\hline SumRelativeAngles & 1 & 282.22 & 282.22 & 5000 & $<2 \mathrm{e}-16^{* * *}$ \\
\hline StandardDeviationAbsoluteAngle & 1 & 431.80 & 431.80 & 5000 & $<2 \mathrm{e}-16^{* * *}$ \\
\hline Residuals & 17 & 320.76 & 18.87 & & \\
\hline
\end{tabular}

Fig. 2. One output of the aovp() function.

\section{Conclusion}

Regarding our preliminary results in Figure 2, it seems that a link exists between some gaze metrics and the global score obtained at the Esperanto exam. These metrics are promising to predict the global learning quality. However, we found out that other combinations of gaze characteristics actually explain this variable. As a perspective, our ambition is to identify, map and order all gaze parameters based on their ability to predict the quality of the recall memory. In addition, beyond the global memorization score, we aim at developing a machine learning technique that can accurately distinguish what is learned from what is forgotten. We plan to extend this pilot study by passing our study to entire classes in our academy, so as to increase the statistic power of our tests. We hope that these preliminary study is a first step toward recommender systems based on the memory of users. 


\section{References}

1. Aben, B., Stapert, S., Blokland, A.: About the distinction between working memory and short-term memory. Frontiers in Psychology 3, 301 (2012)

2. Baddeley, A.: The episodic buffer: a new component of working memory? Trends in Cognitive Sciences 4(11), $417-423$ (2000). https://doi.org/https://doi.org/10.1016/S1364-6613(00)01538-2

3. Bondareva, D., Conati, C., Feyzi-Behnagh, R., Harley, J.M., Azevedo, R., Bouchet, F.: Inferring learning from gaze data during interaction with an environment to support self-regulated learning. In: Lane, H.C., Yacef, K., Mostow, J., Pavlik, P. (eds.) Artificial Intelligence in Education. pp. 229-238. Springer Berlin Heidelberg, Berlin, Heidelberg (2013)

4. Borkin, M.A., Bylinskii, Z., Kim, N.W., Bainbridge, C.M., Yeh, C.S., Borkin, D., Pfister, H., Oliva, A.: Beyond Memorability: Visualization Recognition and Recall. IEEE Transactions on Visualization and Computer Graphics 22(1), 519-528 (2016)

5. Bylinskii, Z., Borkin, M.A., Kim, N.W., Pfister, H., Oliva, A.: Eye fixation metrics for large scale evaluation and comparison of information visualizations. In: Burch, M., Chuang, L., Fisher, B., Schmidt, A., Weiskopf, D. (eds.) Eye Tracking and Visualization. pp. 235-255. Springer International Publishing, Cham (2017)

6. Clariana, R.B., Lee, D.: The effects of recognition and recall study tasks with feedback in a computer-based vocabulary lesson. Educational Technology Research and Development 49(3), 23-36 (2001)

7. Hannula, D.E., Ranganath, C.: The eyes have it: Hippocampal activity predicts expression of memory in eye movements. Neuron 63, 592-599 (2009)

8. Holland, C., Komogortsev, O.V.: Biometric identification via eye movement scanpaths in reading. In: 2011 International Joint Conference on Biometrics (IJCB). pp. 1-8 (Oct 2011). https://doi.org/10.1109/IJCB.2011.6117536

9. Just, M.A., Carpenter, P.A.: Eye fixations and cognitive processes. Cognitive Psychology 8, 441-480 (1976)

10. Komogortsev, O., Gobert, D., Jayarathna, S., Koh, D., Gowda, S.: Standardization of automated analyses of oculomotor fixation and saccadic behaviors. Biomedical Engineering, IEEE Transactions on 57, 2635 - 2645 (12 2010). https://doi.org/10.1109/TBME.2010.2057429

11. Komogortsev, O.V., Jayarathna, S., Koh, D.H., Gowda, S.M.: Qualitative and quantitative scoring and evaluation of the eye movement classification algorithms. In: Proceedings of the 2010 Symposium on EyeTracking Research \&\#38; Applications. pp. 65-68. ETRA '10, ACM, New York, NY, USA (2010). https://doi.org/10.1145/1743666.1743682, http://doi.acm.org/10.1145/1743666.1743682

12. M. Shiffrin, R., C. Atkinson, R.: Storage and retrieval processes in long-term memory. Psychological Review 76, 179-193 (03 1969). https://doi.org/10.1037/h0027277

13. Marchal, F., Castagnos, S., Boyer, A.: First attempt to predict user memory from gaze data. International Journal on Artificial Intelligence Tools 27(6) (2018)

14. Miyake, A., Friedman, N., J. Emerson, M., Witzki, A., Howerter, A., Wager, T.: The unity and diversity of executive functions and their contributions to complex frontal lobe tasks: A latent variable analysis. Cognitive psychology 41, 49-100 (09 2000). https://doi.org/10.1006/cogp.1999.0734

15. Salvucci, D.D., Goldberg, J.H.: Identifying fixations and saccades in eye-tracking protocols. In: Proceedings of the 2000 Symposium on 
Eye Tracking Research \& Applications. pp. 71-78. ETRA '00, ACM, New York, NY, USA (2000). https://doi.org/10.1145/355017.355028, http://doi.acm.org/10.1145/355017.355028

16. Sharafi, Z., Shaffer, T., Sharif, B., Guhneuc, Y.: Eye-tracking metrics in software engineering. In: 2015 Asia-Pacific Software Engineering Conference (APSEC). pp. 96-103 (Dec 2015). https://doi.org/10.1109/APSEC.2015.53

17. Squire, L.R., Dede, A.J.O.: Conscious and unconscious memory systems. Cold Spring Harbor perspectives in biology 7 3, a021667 (2015)

18. Steichen, B., Wu, M.M., Toker, D., Conati, C., Carenini, G.: Te, Te, Hi, Hi: Eye gaze sequence analysis for informing user-adaptive information visualizations. In: User Modeling, Adaptation, and Personalization. pp. 183-194 (2014) 